\title{
Weak and strong deflection gravitational lensing by a renormalization group improved Schwarzschild black hole
}

\author{
$\mathbf{X u} \mathbf{L u}^{1,2}, \mathbf{Y i ~ X i e}^{1,2, \mathrm{a}}$ \\ ${ }^{1}$ School of Astronomy and Space Science, Nanjing University, Nanjing 210023, China \\ ${ }^{2}$ Key Laboratory of Modern Astronomy and Astrophysics, Ministry of Education, Nanjing University, Nanjing 210023, China
}

Received: 29 July 2019 / Accepted: 6 December 2019 / Published online: 17 December 2019

(c) The Author(s) 2019

\begin{abstract}
Weak and strong deflection gravitational lensing by a renormalization group improved Schwarzschild black hole is investigated and its observables are found. By taking the supermassive black holes Sgr A* and M87* respectively in the Galactic Center and at the center of M87 as lenses, we estimate these observables and analyse possibility of detecting this quantum improvement. It is not feasible to distinguish such a black hole by most observables in the near future except for the apparent size of the shadow. We also note that directly using measured shadow of M87* to constrain this quantum effect requires great care.
\end{abstract}

\section{Introduction}

Black holes have been found to be very common in the Universe by direct detections of their gravitational waves [1-6] and by directly imaging the shadow of M87*, the supermassive black hole in the center of galaxy M87 [7-12]. Einstein's general relativity (GR) passes strong-field gravitational tests imposed by these observations. Even though GR succeeds in the vicinities of black holes, it fails in the center of a black hole, meeting the singularity. It is widely believed that such a singularity can only be erased by a quantum theory of gravity because there has exceedingly high energy density and curvature [13]. In order to fulfill this purpose before a self-consistent and well-accepted quantum theory of gravity emerges, one might change the singularity into a regular core [14-17], make use of the quantum pressure to stop collapse of matter and to cause a bounce [18-21], and remove event horizons by creating a quasi-black hole [22-27] (see Ref. [28] for a review). Therefore, a black hole might be an intrinsically quantum object so that quantum gravitational effects might somehow manifest themselves near the event horizons [29-32] because of quantum fluctuations [33,34], a

\footnotetext{
a e-mail: yixie@nju.edu.cn
}

fuzzball $[35,36]$ and an exotic compact object $[37,38]$, which may solve the information loss paradox.

We focus on a renormalization group improved Schwarzschild black hole [39]. Its key idea is to "renormalization group improve" the Schwarzschild spacetime by using the running Newton constant, borrowed from a standard scheme in the particle physics. A benefit from this is the removal of the classical singularity at the center of a Schwarzschild black hole and replacement of it with a de Sitter core by the quantum effects. Its characteristics of black hole physics, such as number of horizons, critical mass, regularity and thermodynamics [39], as well as its quantum gravitational effects on accretion [40] have been studied, whereas its gravitational lensing signatures are still missing in the literature.

A large amount of insights about black holes can be provided by gravitational lensing [41]. With bending angle much smaller than 1, weak deflection lensing has already been a workhorse in astronomy [42-45] and gravitational physics [46-51]. With a key ingredient that a photon might be able to go around a black hole by at least one loop, strong deflection lensing can form an escape cone of light [52] (now popularly called "shadow") and relativistic images [53] (see Refs. [54,55] for reviews). The Event Horizon Telescope (EHT) has directly imaged the shadow of M87* and measured its diameter as 42 microarcsecond ( $\mu$ as) [7-12]. It is also directly imaging the shadow of Sgr $\mathrm{A}^{*}$, the supermassive black hole in the Galactic Center, and its results may be coming soon. Relativistic images might potentially be observable in the future and be helpful to understand nature of black holes [56-60] and distinguish different kinds of them [6165].

Gravitational lensing by quantum-corrected black holes has been studied. Weak deflection lensing by a quantum perturbed lukewarm black hole with a cosmological constant was investigated [66]. A lukewarm black hole belongs to a particular class of Reissner-Nordström-de Sitter solution 
with its electrical charge equal to its mass [67], whereas the renormalization group improved Schwarzschild black hole is neutral. It might be very unlikely to find an electric charged black hole in the real universe since any charge would be neutralized by surrounding plasma. Strong deflection lensing by a non-commutative Schwarzschild black hole was examined [68]. Its spacetime depends on the lower incomplete Gamma function and its non-commutativity is controlled by a new fundamental natural length scale which should be less than $10^{-18} \mathrm{~m}$ because this quantum correction is not visible at presently accessible energies [69]. These are its two different properties from the quantum corrected black hole discussed in this work. The weak and strong deflection lensing has also been used to probe regular black holes, such as Bardeen [70-72], Hayward [73], modified Hayward [74], Lee-Wick [75] and non-minimal Einstein-Yang-Mills black holes [76]. All of these regular black holes have de-Sitter cores, while they have different exterior spacetime due to various physical origins. The renormalization group improved Schwarzschild black hole has its own quantum correction on the exterior spacetime, which is different from those of all aforementioned regular black holes (see below for details) and would generate different gravitational lensing signatures.

Motivated by these considerations, we will investigate the weak and strong deflection gravitational lensing by the renormalization group improved Schwarzschild black hole in the present work. In order to fully understand its lensing signatures, it is necessary to combine these two complementary kinds of lensing, providing a whole picture [77-82].

In Sect. 2, the spacetime of the renormalization group improved Schwarzschild black hole is briefly recalled and a generic description for the gravitational lensing is given. The weak and strong deflection lensing by such a quantum improved black hole is respectively studied in Sects. 3 and 4. By taking Sgr A* and M87* as lenses, we estimate their observables and assess possibility of detecting them. We conclude and discuss our results in Sect. 5.

\section{Metric and gravitational lensing}

\subsection{Metric}

The spacetime of the renormalization group improved Schwarzschild black hole with mass $m_{\bullet}$ is [39]

$\mathrm{d} s^{2}=-A(r) \mathrm{d} t^{2}+B(r) \mathrm{d} r^{2}+C(r)\left(\mathrm{d} \theta^{2}+\sin ^{2} \theta \mathrm{d} \varphi^{2}\right)$,

where the coefficients $A(r), B(r)$ and $C(r)$ are

$$
\begin{aligned}
A(r) & =[B(r)]^{-1} \\
& =1-\frac{2 G m_{\bullet}}{c^{2} r}\left(1+\frac{\tilde{\omega} G \hbar}{c^{3} r^{2}}+\gamma \frac{\tilde{\omega} G^{2} \hbar m_{\bullet}}{c^{5} r^{3}}\right)^{-1}
\end{aligned}
$$

and

$C(r)=r^{2}$

Here, $\gamma$ and $\tilde{\omega}$ are dimensionless parameters respectively from an identification of cutoff of the distance scale and from the nonperturbative renormalization group theory. When the renormalization group improvement vanishes, both of them become zeros. While $\gamma$ might be fixed as $9 / 2$, it can be treated as a positive free parameter [39]. Although $\tilde{\omega}$ might also be determined by comparing the semi-classical Newtonian potential $(A-1) / 2$ with a quantum corrected Newtonian potential, its value in the literature is not unique, such as $\tilde{\omega}=118 /(15 \pi)$ [83] or $\tilde{\omega}=167 /(30 \pi)$ [84] (see Table 1 in [85] for a summary but with a different notation), so that it is likewise treated as a positive free parameter. Such a black hole (1) is regular with a de Sitter core as $r \rightarrow$ 0 . Recently, shadow casted by an improved Schwarzschild spacetime inspired by asymptotically safe quantum gravity was studied [86] whereas its quantum correction is in the form of $\left[1+\mathcal{O}\left(r^{-3}\right)\right]^{-1}$ without the $\mathcal{O}\left(r^{-2}\right)$ term in Eq. (2).

For convenience, convention of $G=c=\hbar=1$ will be adopted in the following parts of this work and it is defined that

$\Omega=\frac{\tilde{\omega}}{m_{\bullet}^{2}}$

which leads to

$A(r)=[B(r)]^{-1}=1-\frac{2 m_{\bullet}}{r}\left(1+\Omega \frac{m_{\bullet}^{2}}{r^{2}}+\gamma \Omega \frac{m_{\bullet}^{3}}{r^{3}}\right)^{-1}$.

It was found [39] that the renormalization group improved Schwarzschild black hole might have none, one or two event horizon(s). The existence of the event horizon(s) that $A(r)=$ 0 gives a cubic equation of $r$ as

$r^{3}-2 m_{\bullet} r^{2}+\Omega m_{\bullet}^{2} r+\gamma \Omega m_{\bullet}^{3}=0$

and its discriminant is

$\Delta_{3}=-m_{\bullet}^{6} \Omega\left(\Omega-\Omega_{+}\right)\left(\Omega-\Omega_{-}\right)$

where

$\Omega_{ \pm}=-\frac{27}{8} \gamma^{2}-\frac{9}{2} \gamma+\frac{1}{2} \pm \frac{1}{8} \sqrt{(\gamma+2)(9 \gamma+2)^{3}}$.

Descartes' rule of signs tells that such a cubic equation has either two positive roots or none because of $\gamma>0$ and $\Omega>$ 0 . In order to ensure $\Delta_{3} \geq 0$, we must have $\Omega \leq \Omega_{+}$given $\Omega_{-}<0$ so that we define a dimensionless parameter $\lambda$ as 
$\lambda=\frac{\Omega}{\Omega_{+}} \in(0,1]$.

It means that any $\Omega$ can be uniquely determined by $\lambda$ and $\gamma$ because $\Omega_{+}$is a function of $\gamma$ [see Eq. (8)], i.e., $\Omega=$ $\lambda \Omega_{+}(\gamma)$. Summarily, when $0<\lambda<1$, two event horizons exist; when $\lambda=1$, they merger into one; when $\lambda>1$, there is no event horizon. The (outer) event horizon decreases to its smallest value $r_{\mathrm{H}, \min }=m_{\bullet}$ at $\lambda=1$ and $\gamma=0$. Finally, the parameter space of the renormalization improved Schwarzschild black hole is set as $\mathcal{D}=\{(\gamma, \lambda) \mid 0<\gamma \leq$ $10,0<\lambda \leq 1\}$ in which an upper-bound on $\gamma$ is chosen and $\Omega_{+}(\gamma)>0$ for $\gamma \in \mathcal{D}$.

\subsection{Gravitational lensing}

It is necessary to understand observational signatures of the renormalization group improved Schwarzschild black hole before searching and distinguishing them. Since groundbased infrared interferometry has been routinely monitoring Sgr A* and stars near it [87-89] and ground-based radio interferometry has successfully image the shadow of M87* [7-12], we aim at studying gravitational lensing by the supermassive black holes with this renormalization group improvement.

The exact bending angle in the gravitational lensing by an asymptotically flat and spherically symmetric black hole is well known as $[56,90]$

$\hat{\alpha}\left(r_{0}\right)=2 \int_{r_{0}}^{\infty} \frac{\sqrt{B(r)}}{\sqrt{C(r)} \sqrt{\frac{C(r)}{C\left(r_{0}\right)} \frac{A\left(r_{0}\right)}{A(r)}-1}} \mathrm{~d} r-\pi$,

where $r_{0}$ is the closet approach distance of the light ray to the black hole. In the weak deflection lensing, $r_{0}$ is much bigger than $m_{\bullet}$, resulting $\hat{\alpha} \ll 1$. In the strong deflection lensing, $r_{0}$ is approaching to $\sim m_{\bullet}$, making $\hat{\alpha} \gg 1$.

Finding observables of gravitational lensing needs the lens equation. It is adopted that $[56,57]$

$\tan \mathcal{B}=\tan \vartheta-\frac{D_{\mathrm{LS}}}{D_{\mathrm{OS}}}[\tan \vartheta+\tan (\hat{\alpha}-\vartheta)]$,

where $\mathcal{B}$ is the angular position of the source, $\vartheta$ is the angular position of the image, and $D_{\mathrm{LS}}$ and $D_{\mathrm{OS}}$ are the projected angular diameter distances on the optical axis respectively from the lens to the source and from the observer to the source. The signed magnification $\mu$ of a lensed image is [91]

$\mu(\vartheta)=\left[\frac{\sin \mathcal{B}(\vartheta)}{\sin \vartheta} \frac{\mathrm{d} \mathcal{B}(\vartheta)}{\mathrm{d} \vartheta}\right]^{-1}$

If a source's luminosity evolves, it would be able to measure time delay between its lensed images. The time delay is directly relevant to the flight time taken by a photon from the source to the observer $[90,92,93]$

$T=T\left(R_{\mathrm{src}}\right)+T\left(R_{\mathrm{obs}}\right)$,

with

$T(R)=\int_{r_{0}}^{R}\left|\frac{\mathrm{d} t}{\mathrm{~d} r}\right| \mathrm{d} r$

and

$\frac{\mathrm{d} t}{\mathrm{~d} r}=\frac{\sqrt{B(r) C(r) A\left(r_{0}\right)}}{A(r) \sqrt{C\left(r_{0}\right)} \sqrt{\frac{C(r)}{C\left(r_{0}\right)} \frac{A\left(r_{0}\right)}{A(r)}-1}}$,

where $R_{\mathrm{obs}}$ is the distance from the lens to the observer, i.e. $R_{\mathrm{obs}}=D_{\mathrm{OL}}$, and $R_{\mathrm{src}}$ is the radial coordinate of the source with respect to the lens, i.e. $R_{\text {src }}=\left(D_{\text {OS }}^{2} \tan ^{2} \mathcal{B}+D_{\mathrm{LS}}^{2}\right)^{1 / 2}$.

In each scenario of weak and strong deflection lensing, we will investigate its bending angle, lens equation, magnification, time delay and their observables for Sgr A* and M87*.

\section{Weak deflection lensing}

\subsection{Bending angle}

In the spacetime (1), the distance of closet approach $r_{0}$ for the light ray relates with its impact parameter $u$ via the relation as [90]

$C\left(r_{0}\right)=u^{2} A\left(r_{0}\right)$.

Since both $r_{0}$ and $u$ are much larger than $m_{\bullet}$ in the weak deflection lensing, we can expand the solution to (16) in terms of $q \equiv m \bullet u^{-1}$ as

$\frac{r_{0}}{u}=1+\sum_{n=1}^{6} \mathfrak{u}_{n} q^{n}+\mathcal{O}\left(q^{7}\right)$

where

$$
\begin{aligned}
& \mathfrak{u}_{1}=-1, \\
& \mathfrak{u}_{2}=-\frac{3}{2}, \\
& \mathfrak{u}_{3}=\Omega-4, \\
& \mathfrak{u}_{4}=(\gamma+5) \Omega-\frac{105}{8}, \\
& \mathfrak{u}_{5}=-\Omega^{2}+6(\gamma+4) \Omega-48, \\
& \mathfrak{u}_{6}=-\frac{1}{2}(4 \gamma+21) \Omega^{2}+\frac{21}{2}(3 \gamma+11) \Omega-\frac{3003}{16} .
\end{aligned}
$$


The bending angle in the weak deflection lensing can be found in the form of a series according to $h \equiv m_{\bullet} r_{0}^{-1}$ as

$\hat{\alpha}(h)=\sum_{n=1}^{6} \mathfrak{a}_{n} h^{n}+\mathcal{O}\left(h^{7}\right)$

with

$$
\begin{aligned}
\mathfrak{a}_{1}= & 4, \\
\mathfrak{a}_{2}= & \frac{15}{4} \pi-4, \\
\mathfrak{a}_{3}= & -\frac{16}{3} \Omega-\frac{15}{2} \pi+\frac{122}{3}, \\
\mathfrak{a}_{4}= & -\left(\frac{15}{8} \gamma \pi+\frac{105}{8} \pi-20\right) \Omega+\frac{3465}{64} \pi-130, \\
\mathfrak{a}_{5}= & \frac{32}{5} \Omega^{2}+\left[\left(\frac{15}{2} \pi-\frac{236}{5}\right) \gamma+60 \pi-260\right] \Omega \\
& -\frac{3465}{16} \pi+\frac{7783}{10}, \\
\mathfrak{a}_{6}= & \left(\frac{35}{8} \gamma \pi+\frac{945}{32} \pi-52\right) \Omega^{2} \\
& +\left[\left(260-\frac{3465}{32} \pi\right) \gamma-\frac{16695}{32} \pi+\frac{4234}{3}\right] \Omega \\
& +\frac{310695}{256} \pi-\frac{21397}{6} .
\end{aligned}
$$

Its dependence of the coordinate $r_{0}$ makes the expression gauge-dependent. Replacing $r_{0}$ with the impact parameter $u$ by using Eq. (17), we are able to obtain its gauge-invariant form as

$\hat{\alpha}(u)=\sum_{n=1}^{6} \hat{\mathfrak{u}}_{n} q^{n}+\mathcal{O}\left(q^{7}\right)$,

in which

$\hat{\mathfrak{u}}_{1}=4$,

$\hat{\mathfrak{u}}_{2}=\frac{15}{4} \pi$,

$\hat{\mathfrak{u}}_{3}=-\frac{16}{3}(\Omega-8)$,

$\hat{\mathfrak{u}}_{4}=-\frac{15}{8} \pi\left[(\gamma+7) \Omega-\frac{231}{8}\right]$,

$\hat{\mathfrak{u}}_{5}=\frac{32}{5} \Omega^{2}-\frac{256}{5}(\gamma+5) \Omega+\frac{3584}{5}$,

$\hat{\mathfrak{u}}_{6}=\frac{35}{256} \pi\left[8(4 \gamma+27) \Omega^{2}-264(3 \gamma+13) \Omega+7293\right]$.

When $\Omega=\gamma=0$, the bending angle in the either gaugedependent or gauge-invariant form returns to its accordingly value for the Schwarzschild black hole in GR. It is obvious that the renormalization group improvement begins to affect the bending angle at the third-order approximation.

\subsection{Lens equation}

For later convenience, some scaled variables are defined as [46-48]

$\beta=\frac{\mathcal{B}}{\vartheta_{\mathrm{E}}}, \quad \theta=\frac{\vartheta}{\vartheta_{\mathrm{E}}}, \quad \hat{\tau}=\frac{\tau}{\tau_{\mathrm{E}}}, \quad \varepsilon=\frac{\vartheta_{\bullet}}{\vartheta_{\mathrm{E}}}$,

where $\vartheta_{\bullet}=\arctan \left(m_{\bullet} / D_{\mathrm{OL}}\right)$ is the angular gravitational radius at distance $D_{\mathrm{OL}}, \vartheta=\arcsin \left(u / D_{\mathrm{OL}}\right), \tau$ is the time delay between images, the angular Einstein ring radius is

$\vartheta_{\mathrm{E}}=\sqrt{\frac{4 m_{\bullet} D_{\mathrm{LS}}}{D_{\mathrm{OL}} D_{\mathrm{OS}}}}$

and the time scale is

$\tau_{\mathrm{E}}=4 m_{\bullet}$

In the weak deflection gravitational lensing, $\varepsilon$ can be considered as a small parameter as long as both the observer and the source are far enough from the lens, and will be employed to find out higher-order observables for the renormalization group improved Schwarzschild black hole which might be the only signals influenced by the quantum correction.

With the help of $\varepsilon$, it is reasonable to expand the solution to the lens Eq. (11) into a series as

$\theta=\theta_{0}+\varepsilon \theta_{1}+\varepsilon^{2} \theta_{2}+\mathcal{O}\left(\varepsilon^{3}\right)$

where $\theta_{0}, \theta_{1}$ and $\theta_{2}$ are its zeroth-, first- and second-order approximation terms. Therefore, the bending angle (30) might have the form as

$$
\begin{aligned}
\hat{\alpha}= & 4 \frac{\varepsilon}{\theta_{0}}+\frac{\varepsilon^{2}}{\theta_{0}^{2}}\left(\frac{15}{4} \pi-4 \theta_{1}\right)+\frac{\varepsilon^{3}}{\theta_{0}^{3}}\left\{\frac{32}{3} D^{2} \theta_{0}^{4}\right. \\
& \left.-4 \theta_{0} \theta_{2}+4 \theta_{1}^{2}-\frac{15}{2} \pi \theta_{1}-\frac{16}{3} \Omega+\frac{128}{3}\right\} \\
& +\mathcal{O}\left(\varepsilon^{4}\right),
\end{aligned}
$$

and the lens Eq. (11) might be transformed into

$$
\begin{aligned}
0= & 4 D \frac{\varepsilon}{\theta_{0}}\left(\beta \theta_{0}-\theta_{0}^{2}+1\right)+\frac{\varepsilon^{2}}{\theta_{0}^{2}} D\left[\frac{15}{4} \pi-4 \theta_{1}\left(\theta_{0}^{2}+1\right)\right] \\
& +\frac{\varepsilon^{3}}{\theta_{0}^{3}} D\left[-\frac{64}{3} D^{2} \theta_{0}^{6}+\frac{224}{3} D^{2} \theta_{0}^{4}+\left(\frac{64}{3} D^{2} \beta^{3}\right.\right. \\
& \left.-4 \theta_{2}\right) \theta_{0}^{3}-64 D \theta_{0}^{2}-4 \theta_{0} \theta_{2}+4 \theta_{1}^{2}-\frac{15}{2} \pi \theta_{1} \\
& \left.-\frac{16}{3} \Omega+64\right]+\mathcal{O}\left(\varepsilon^{4}\right),
\end{aligned}
$$


where $D=D_{\mathrm{LS}} / D_{\mathrm{OS}}$. This lens equation gives positions of the lensed images by orders of $\varepsilon$.

\subsection{Image positions}

$\theta_{n}(n=0,1,2)$ can be calculated by letting the coefficients of $\epsilon, \epsilon^{2}$ and $\epsilon^{3}$ in (42) equal to 0 . For the zeroth-order image position, it leads to

$\beta=\theta_{0}-\frac{1}{\theta_{0}}$,

resulting

$\theta_{0}=\frac{1}{2}(\beta+\eta)$

where

$\eta=\sqrt{\beta^{2}+4}$

Similarly, we can find the first- and second-order corrections to the image position as

$$
\begin{aligned}
\theta_{1}= & \frac{15}{16} \frac{\pi}{\theta_{0}^{2}+1}, \\
\theta_{2}= & \frac{1}{\theta_{0}\left(\theta_{0}^{2}+1\right)^{3}}\left[\frac{8}{3} D^{2} \theta_{0}^{8}+D\left(\frac{64}{3} D-16\right) \theta_{0}^{6}\right. \\
& +\left(\frac{88}{3} D^{2}-32 D-\frac{4}{3} \Omega+16\right) \theta_{0}^{4} \\
& +\left(\frac{16}{3} D^{2}-16 D-\frac{8}{3} \Omega+32-\frac{225}{128} \pi^{2}\right) \theta_{0}^{2} \\
& -\frac{16}{3} D^{2}-\frac{4}{3} \Omega-\frac{225}{256} \pi^{2}+16 .
\end{aligned}
$$

The zeroth- and first-order approximation terms are as the same as those of the Schwarzschild black hole in GR [46,47] free of the quantum correction, while the second-order term is affected by renormalization group improvement if $\Omega \neq 0$.

\subsection{Magnifications}

The magnification $\mu$ of a lensed image can also be expanded in terms of $\epsilon$ as a series

$\mu=\mu_{0}+\varepsilon \mu_{1}+\varepsilon^{2} \mu_{2}+\mathcal{O}\left(\varepsilon^{3}\right)$,

where the zeroth-, first- and second-order terms are

$$
\begin{aligned}
& \mu_{0}=\frac{\theta_{0}^{4}}{\theta_{0}^{4}-1}, \\
& \mu_{1}=-\frac{15}{16} \pi \frac{\theta_{0}^{3}}{\left(\theta_{0}^{2}+1\right)^{3}},
\end{aligned}
$$

$$
\begin{aligned}
\mu_{2}= & \frac{\theta_{0}^{2}}{\left(\theta_{0}^{2}+1\right)^{5}\left(\theta_{0}^{2}-1\right)}\left[\frac{8}{3} D^{2} \theta_{0}^{8}\right. \\
& +\left(48 D^{2}-32 D+\frac{8}{3} \Omega-32\right) \theta_{0}^{6} \\
& +\left(\frac{272}{3} D^{2}-64 D+\frac{16}{3} \Omega+\frac{675}{128} \pi^{2}-64\right) \theta_{0}^{4} \\
& \left.+\left(48 D^{2}-32 D+\frac{8}{3} \Omega-32\right) \theta_{0}^{2}+\frac{8}{3} D^{2}\right] .
\end{aligned}
$$

Like the situation of the images, the zeroth- and first-order term are immune to the quantum correction, whereas the second-order one is not. When $\Omega=0$, the magnification $\mu$ reduces to the one of the Schwarzschild black hole [46,47].

\subsection{Time delay}

The flight time function $T(R)$ can be expanded in terms of $h$ as

$T(R)=T_{0}+r_{0} \sum_{n=1}^{3} T_{n} h^{n}+\mathcal{O}\left(h^{4}\right)$,

where its zeroth-, first-, second- and third-order approximation terms are

$$
\begin{aligned}
T_{0}= & \sqrt{R^{2}-r_{0}^{2}}, \\
T_{1}= & \frac{\sqrt{1-\xi^{2}}}{1+\xi}+2 \ln \left(\frac{1+\sqrt{1-\xi^{2}}}{\xi}\right) \\
T_{2}= & \frac{15}{4} \pi-\frac{15}{2} \arctan \left(\frac{\xi}{\sqrt{1-\xi^{2}}}\right) \\
& -\frac{\sqrt{1-\xi^{2}}}{(1+\xi)^{2}}\left(\frac{5}{2} \xi+2\right) \\
T_{3}= & -\frac{15}{4} \pi+\frac{15}{2} \arctan \left(\frac{\xi}{\sqrt{1-\xi^{2}}}\right) \\
& +\frac{\sqrt{1-\xi^{2}}}{2(1+\xi)^{3}}\left(35 \xi^{3}+133 \xi^{2}+157 \xi+60\right) \\
& -\frac{\sqrt{1-\xi^{2}}}{1+\xi}(3 \xi+4) \Omega
\end{aligned}
$$

with

$\xi=\frac{r_{0}}{R}$.

It is at the third-order term of $T(R)$ that the renormalization group improvement begins to manifest. When $\Omega$ vanishes, $T(R)$ returns to the one of the Schwarzschild black hole [46, 47].

The difference between the flight time of the light with and without gravitational influence of the lens is defined as 
the differential time delay which is mathematically expressed as

$c \tau=T\left(R_{\mathrm{src}}\right)+T\left(R_{\mathrm{obs}}\right)-\frac{D_{\mathrm{OS}}}{\cos \mathcal{B}}$.

Making use of the relation between $r_{0}$ and $u$ and expressions for $R_{\mathrm{src}}$ and $R_{\mathrm{obs}}$, we can find the scaled time delay in the form of a series of $\varepsilon$ as

$\hat{\tau}=\hat{\tau}_{0}+\varepsilon \hat{\tau}_{1}+\varepsilon^{2} \hat{\tau}_{2}+\mathcal{O}\left(\varepsilon^{3}\right)$,

where

$$
\begin{aligned}
\hat{\tau}_{0}= & \frac{1}{2}\left[1+\beta^{2}-\theta_{0}^{2}-\ln \left(\frac{D_{\mathrm{OL}} \theta_{0}^{2} \vartheta_{\mathrm{E}}^{2}}{4 D_{\mathrm{LS}}}\right)\right] \\
\hat{\tau}_{1}= & \frac{15 \pi}{16 \theta_{0}} \\
\hat{\tau}_{2}= & \frac{1}{2 \theta_{0}^{2}}\left\{-\frac{4}{3} \theta_{0}^{6}\left(5 D^{2}-9 D+3\right)-8 \theta_{0}^{4} \beta^{2}(D-1)\right. \\
& +\frac{4}{3} \theta_{0}^{2}\left[\left(5 D^{2}-3 D-3\right) \beta^{4}+6 \beta^{2}-6-24 D^{2}+24 D\right] \\
& \left.-\frac{225}{256} \frac{\pi^{2}}{\theta_{0}^{2}+1}+\frac{32}{3} D^{2}-\frac{4}{3} \Omega\right\}
\end{aligned}
$$

When $\Omega=0$, the scaled time delay is the same as the one of the Schwarzschild black hole $[46,47]$. The quantum correction only affect its second-order approximation term.

\subsection{Relations}

Hereafter, following Refs. [46-48], the convention that the angle of an image's position is always positive is adopted. If the image is on the same side of the lens as the source, the position of the source $\mathcal{B}$ is positive; otherwise, $\mathcal{B}<0$.

\subsubsection{Position relations}

After picking $\beta>0$ and $\beta<0$, the positive- and negativeparity images at the leading order [see Eq. (44)] can be found as

$\theta_{0}^{ \pm}=\frac{1}{2}(\eta \pm|\beta|)$

which result in

$$
\begin{aligned}
\theta_{0}^{+}-\theta_{0}^{-} & =|\beta|, \\
\theta_{0}^{+} \theta_{0}^{-} & =1 .
\end{aligned}
$$

With $\theta_{0}^{ \pm}$, the first-order corrections to the image positions can be obtained as

$\theta_{1}^{ \pm}=\frac{15}{8} \frac{\pi}{\eta(\eta \pm|\beta|)}$, which can be combined as

$\theta_{1}^{+}+\theta_{1}^{-}=\frac{15}{16} \pi$,

$\theta_{1}^{+}-\theta_{1}^{-}=-\frac{15}{16} \pi \frac{|\beta|}{\eta}$.

Furthermore, the second-order corrections to the image positions can be found as

$\theta_{2}^{ \pm}=\mathfrak{P}_{ \pm}\left(\mathfrak{P} \pm \mathfrak{P}^{\prime} \eta|\beta|\right)$,

where the factors $\mathfrak{P}_{ \pm}$and the coefficients of $\mathfrak{P}$ and $\mathfrak{P}^{\prime}$ are

$$
\begin{aligned}
\mathfrak{P}_{ \pm}= & \frac{1}{\eta^{3}(\eta \pm|\beta|)^{4}}, \\
\mathfrak{P}= & \frac{64}{3} D^{2} \beta^{8}+D\left(\frac{1024}{3} D-128\right) \beta^{6} \\
& +\left(\frac{5056}{3} D^{2}-1024 D+128\right) \beta^{4} \\
& +\left(\frac{8576}{3} D^{2}-2304 D+768-\frac{225}{16} \pi^{2}\right) \beta^{2} \\
& +\frac{2560}{3} D^{2}-1024 D+1024-\frac{675}{16} \pi^{2} \\
& -\frac{32}{3}\left(\beta^{2}+2\right) \eta^{2} \Omega, \\
\mathfrak{P}^{\prime}= & \frac{64}{3} D^{2} \beta^{6}+D\left(\frac{896}{3} D-128\right) \beta^{4} \\
& +\left(\frac{3392}{3} D^{2}-768 D+128\right) \beta^{2} \\
& +\frac{3328}{3} D^{2}-1024 D+512-\frac{225}{16} \pi^{2} \\
& -\frac{32}{3} \eta^{2} \Omega .
\end{aligned}
$$

They have a simple relation that

$\theta_{2}^{+}-\theta_{2}^{-}=|\beta|\left(8 D^{2}+\frac{225}{256} \pi^{2}-16+\frac{4}{3} \Omega\right)$.

As expected based on the previous results of the images' positions, the renormalization group improvement has only influence on the second-order corrections to the positive- and negative-parity of images and their relations. When $\Omega=0$, they all go back to those of the Schwarzschild black hole $[46,47]$.

\subsubsection{Magnification relations}

Using $\mu_{n}(n=0,1,2)$ for the magnifications and $\theta_{0}^{ \pm}$for the images' positions, we can work out

$\mu_{0}^{ \pm}=\frac{1}{2|\beta| \eta}\left[|\beta| \eta \pm\left(\beta^{2}+2\right)\right]$,

$\mu_{1}^{+}=\mu_{1}^{-}=-\frac{15}{16} \frac{\pi}{\eta^{3}}$, 
$\mu_{2}^{ \pm}= \pm \frac{\mathfrak{M}}{|\beta| \eta^{5}}$,

where the factor $\mathfrak{M}$ is

$$
\begin{aligned}
\mathfrak{M}= & \frac{8}{3} D^{2} \beta^{4}+\left(\frac{176}{3} D^{2}-32 D-32\right) \beta^{2} \\
& -128 D+192 D^{2}+\frac{675}{128} \pi^{2}-128+\frac{8}{3} \eta^{2} \Omega .
\end{aligned}
$$

They hold three simple combinations of the magnifications that

$\mu_{0}^{+}+\mu_{0}^{-}=1$,

$\mu_{1}^{+}-\mu_{1}^{-}=0$,

$\mu_{2}^{+}+\mu_{2}^{-}=0$,

and a more complicated but straightforward one

$\mu_{0}^{+} \theta_{1}^{+}+\mu_{0}^{-} \theta_{1}^{-}+\mu_{1}^{+} \theta_{0}^{+}+\mu_{1}^{-} \theta_{0}^{-}=0$.

\subsubsection{Total magnification and centroid}

If the two images in the weak deflection lensing could not be resolved, the total magnification and centroid position would be the observables. The total magnification is

$$
\begin{aligned}
\mu_{\mathrm{tot}} & =\left|\mu^{+}\right|+\left|\mu^{-}\right| \\
& =\left(2 \mu_{0}^{+}-1\right)+2 \epsilon^{2} \mu_{2}^{+}+\mathcal{O}\left(\epsilon^{3}\right) .
\end{aligned}
$$

Because of $\mu_{1}^{+}=\mu_{1}^{-}$, any term at the first-order approximation $\mathcal{O}(\epsilon)$ never appears in $\mu_{\text {tot }}$.

The centroid position is defined as a magnificationweighted summation of the positive- and negative-parity images [46]

$\Theta_{\text {cent }}=\frac{\theta^{+}\left|\mu^{+}\right|-\theta^{-}\left|\mu^{-}\right|}{\left|\mu^{+}\right|+\left|\mu^{-}\right|}=\frac{\theta^{+} \mu^{+}+\theta^{-} \mu^{-}}{\mu^{+}-\mu^{-}}$,

and it can be expanded in terms of $\varepsilon$ into a series as

$\Theta_{\text {cent }}=\Theta_{0}+\varepsilon \Theta_{1}+\varepsilon^{2} \Theta_{2}+\mathcal{O}\left(\varepsilon^{3}\right)$,

where

$$
\begin{aligned}
& \Theta_{0}=|\beta| \frac{\beta^{2}+3}{\beta^{2}+2}, \\
& \Theta_{1}=0, \\
& \Theta_{2}=\frac{|\beta|}{\eta^{2}\left(\beta^{2}+2\right)^{2}} \mathfrak{S},
\end{aligned}
$$

and

$$
\begin{aligned}
\mathfrak{S}= & \frac{8}{3} D^{2} \beta^{6}+\left(\frac{104}{3} D-16\right) D \beta^{4}+\left(\frac{272}{3} D^{2}\right. \\
& -64 D+32) \beta^{2}-\frac{64}{3} D^{2}-\frac{675}{128} \pi^{2}+128
\end{aligned}
$$

$$
-\frac{8}{3} \eta^{2} \Omega
$$

It can be verified that $\mu_{\text {tot }}$ and $\Theta_{\text {cent }}$ reduce to their corresponding values for the Schwarzschild black hole when $\Omega=0[46,47]$; only their second-order approximation terms are affected by the renormalization group improvement.

\subsubsection{Differential time delay}

The differential time delay between the positive- and negativeparity images is

$\Delta \hat{\tau}=\hat{\tau}_{-}-\hat{\tau}_{+}$,

which can also be expanded into a series of $\varepsilon$ as

$\Delta \hat{\tau}=\Delta \hat{\tau}_{0}+\varepsilon \Delta \hat{\tau}_{1}+\varepsilon^{2} \Delta \hat{\tau}_{2}+\mathcal{O}\left(\varepsilon^{3}\right)$,

with

$$
\begin{aligned}
\Delta \hat{\tau}_{0}= & \frac{1}{2} \eta|\beta|+\ln \left(\frac{\eta+|\beta|}{\eta-|\beta|}\right) \\
\Delta \hat{\tau}_{1}= & \frac{15}{16} \pi|\beta| \\
\Delta \hat{\tau}_{2}= & |\beta| \eta\left[-\frac{225}{512} \pi^{2} \frac{\beta^{2}+3}{\eta^{2}}+\left(\frac{10}{3} D^{2}-2 D-2\right) \beta^{2}\right. \\
& \left.+12 D^{2}-12 D+4-\frac{2}{3} \Omega\right]
\end{aligned}
$$

It can likewise be found that the quantum correction only appears in its second-order approximation term and when it vanishes, i.e. $\Omega=0$, the differential time delay will have the same value as the one of the Schwarzschild black hole $[46,47]$.

\subsection{Practical observables}

In practice, observables of the weak deflection lensing are the positions, fluxes and time delays of the lensed images and/or their combinations [47]. Therefore, it is necessary to transform the scaled variables $(\beta, \theta, \mu, \hat{\tau})$ into practical observables $(\mathcal{B}, \vartheta, F, \tau)$, in which the observed flux $F$ is the magnified the one of the source $F_{\text {src }}$ via $F=|\mu| F_{\text {src }}$.

These practical observables and their combinations are

$$
\begin{aligned}
P_{\text {tot }} \equiv & \vartheta^{+}+\vartheta^{-} \\
= & \mathcal{E}+\frac{15}{16} \varepsilon \pi \vartheta_{\mathrm{E}} \\
& +\frac{\varepsilon^{2}}{\mathcal{E}^{3}}\left[-\left(\frac{8}{3} D^{2}+\frac{225}{256} \pi^{2}-16+\frac{4}{3} \Omega\right) \mathcal{B}^{4}\right. \\
& +\left(16 D^{2}-32 D-\frac{675}{128} \pi^{2}+96-8 \Omega\right) \mathcal{B}^{2} \vartheta_{\mathrm{E}}^{2} \\
& +\left(\frac{320}{3} D^{2}-128 D-\frac{675}{128} \pi^{2}+128-\frac{32}{3} \Omega\right) \vartheta_{\mathrm{E}}^{4}
\end{aligned}
$$




$$
\begin{aligned}
& +\mathcal{O}\left(\varepsilon^{3}\right), \\
& \Delta P \equiv \vartheta^{+}-\vartheta^{-}=|\mathcal{B}|\left[1-\frac{15}{16} \varepsilon \pi \frac{\vartheta_{\mathrm{E}}}{\mathcal{E}}\right. \\
& \left.+\varepsilon^{2}\left(8 D^{2}+\frac{225}{256} \pi^{2}-16+\frac{4}{3} \Omega\right)+\mathcal{O}\left(\varepsilon^{3}\right)\right], \\
& F_{\text {tot }} \equiv F^{+}+F^{-} \\
& =\frac{F_{\text {src }}}{|\mathcal{B}| \mathcal{E}}\left\{\mathcal{B}^{2}+2 \vartheta_{\mathrm{E}}^{2}+\frac{\varepsilon^{2}}{\mathcal{E}^{4}}\left[\frac{16}{3} D^{2} \mathcal{B}^{4} \vartheta_{\mathrm{E}}^{2}\right.\right. \\
& +\left(\frac{352}{3} D^{2}-64 D-64+\frac{16}{3} \Omega\right) \mathcal{B}^{2} \vartheta_{\mathrm{E}}^{4} \\
& \left.+\left(384 D^{2}-256 D+\frac{675}{64} \pi^{2}-256+\frac{64}{3} \Omega\right) \vartheta_{\mathrm{E}}^{6}\right] \\
& \left.+\mathcal{O}\left(\varepsilon^{3}\right)\right\}, \\
& \Delta F \equiv F^{+}-F^{-} \\
& =F_{\mathrm{src}}-F_{\mathrm{src}} \frac{15}{8} \varepsilon \pi \frac{\vartheta_{\mathrm{E}}^{3}}{\mathcal{E}^{3}}+\mathcal{O}\left(\varepsilon^{3}\right), \\
& S_{\text {cent }} \equiv \frac{\vartheta^{+} F^{-}-\vartheta^{-} F^{-}}{F_{\text {tot }}} \\
& =|\mathcal{B}| \frac{\mathcal{B}^{2}+3 \vartheta_{\mathrm{E}}^{2}}{\mathcal{B}^{2}+2 \vartheta_{\mathrm{E}}^{2}}+\frac{|\mathcal{B}|}{\mathcal{E}^{2}\left(\mathcal{B}^{2}+2 \vartheta_{\mathrm{E}}^{2}\right)^{2}} \varepsilon^{2}\left[\frac{8}{3} D^{2} \mathcal{B}^{6}\right. \\
& +\left(\frac{104}{3} D-16\right) D \mathcal{B}^{4} \vartheta_{\mathrm{E}}^{2} \\
& +\left(\frac{272}{3} D^{2}-64 D+32\right) \mathcal{B}^{2} \vartheta_{\mathrm{E}}^{4} \\
& \left.+\left(-\frac{64}{3} D^{2}-\frac{675}{128} \pi^{2}+128-\frac{8}{3} \eta^{2} \Omega\right) \vartheta_{\mathrm{E}}^{6}\right] \\
& +\mathcal{O}\left(\varepsilon^{3}\right) \text {, } \\
& \Delta \tau=\frac{D_{\mathrm{OL}} D_{\mathrm{OS}}}{c D_{\mathrm{LS}}}\left\{\frac{1}{2}|\mathcal{B}| \mathcal{E}+\vartheta_{\mathrm{E}}^{2} \ln \left(\frac{\mathcal{E}+|\mathcal{B}|}{\mathcal{E}-|\mathcal{B}|}\right)\right. \\
& +\varepsilon \frac{15}{16} \pi \vartheta_{\mathrm{E}}|\mathcal{B}|+\varepsilon^{2}|\mathcal{B}| \mathcal{E}\left[-\frac{225}{512} \pi^{2} \frac{\mathcal{B}^{2}+3 \vartheta_{\mathrm{E}}^{2}}{\mathcal{E}^{2}}\right. \\
& \left.+\left(\frac{10}{3} D^{2}-2 D-2\right) \frac{\mathcal{B}^{2}}{\vartheta_{\mathrm{E}}^{2}}+12 D(D-1)+4-\frac{2}{3} \Omega\right] \\
& \left.+\mathcal{O}\left(\varepsilon^{3}\right)\right\},
\end{aligned}
$$

where

$$
\mathcal{E}=\sqrt{\mathcal{B}^{2}+4 \vartheta_{\mathrm{E}}^{2}} .
$$

In order to directly show the contributions of the renormalization group improvement in the weak deflection lensing, the deviations of the practical observables from those of the Schwarzschild black hole are defined as

$$
\delta P_{\text {tot }} \equiv P_{\text {tot }}-P_{\text {tot }}(\Omega=0)
$$

$$
\begin{aligned}
& =-\frac{4\left(\beta^{2}+2\right)}{3 \eta} \vartheta_{\mathrm{E}} \varepsilon^{2} \Omega+\mathcal{O}\left(\varepsilon^{3}\right), \\
\delta \Delta P & \equiv \Delta P-\Delta P(\Omega=0)=\frac{4}{3} \varepsilon^{2} \mathcal{B} \Omega+\mathcal{O}\left(\varepsilon^{3}\right), \\
\delta r_{\text {tot }} & \equiv 2.5 \log _{10}\left[\frac{F_{\text {tot }}}{F_{\text {tot }}(\Omega=0)}\right] \\
& =\frac{40}{(3 \ln 10) \eta^{2}\left(\beta^{2}+2\right)} \varepsilon^{2} \Omega+\mathcal{O}\left(\varepsilon^{3}\right), \\
\delta \Delta r & \equiv 2.5 \log _{10}\left[\frac{\Delta F}{\Delta F(\Omega=0)}\right]=\mathcal{O}\left(\varepsilon^{3}\right), \\
\delta S_{\text {cent }} & \equiv S_{\text {cent }}-S_{\text {cent }}(\Omega=0) \\
& =-\frac{8|\mathcal{B}|}{3\left(\beta^{2}+2\right)^{2}} \varepsilon^{2} \Omega+\mathcal{O}\left(\varepsilon^{3}\right), \\
\delta \Delta \tau & \equiv \Delta \tau-\Delta \tau(\Omega=0) \\
& =-\frac{2}{3} \varepsilon^{2} \frac{D_{\mathrm{OL}} D_{\mathrm{OS}}}{c D_{\mathrm{LS}}}|\mathcal{B}| \mathcal{E} \Omega+\mathcal{O}\left(\varepsilon^{3}\right),
\end{aligned}
$$

where the fluxes are changed into the magnitudes of brightness and $\Omega$ can be further expressed as $\Omega=\lambda \Omega_{+}(\gamma)$. It is clear that the differential flux of the two lensed images is not suitable for detecting such a quantum correction because of $\delta \Delta r=\mathcal{O}\left(\varepsilon^{3}\right)$. While the existence of the renormalization group improvement would make $\Delta P$ and $F_{\text {tot }}$ larger than their values of the Schwarzschild black hole $(\Omega=0$ or $\lambda=0$ ), it would cause $P_{\text {tot }}, S_{\text {cent }}$ and $\Delta \tau$ to be smaller.

\subsection{Example of Sgr A*}

Stars orbiting the supermassive black hole Sgr A* have been directly observing and monitoring. We take Sgr A* as the lens with $M=4.28 \times 10^{6} M_{\odot}$ and $D_{\mathrm{OL}}=8.32 \mathrm{kpc}$ [94] and assume a source having a distance $D_{\mathrm{LS}}=10^{-3} \mathrm{pc}$ from it. The Einstein radius of the source $\theta_{\mathrm{E}}$ is 710 microarcsecond ( $\mu$ as) and the small parameter is $\varepsilon=7.2 \times 10^{-3}$. Such an assumption about the source is based on the observed fact that the periastron distance of the star S175 around Sgr A* is about $2 \times 10^{-4} \mathrm{pc}$ [94], five times smaller than the assumed $D_{\mathrm{LS}}$. We note that, for the Schwarzschild black hole in GR, when $\beta=0.5$ in our assumed case, the practical observables for the weak deflection lensing are $P_{\text {tot }}=1.48$ milliarcsecond (mas), $\Delta P=0.35$ mas, $F_{\text {tot }} / F_{\text {src }}=2.18$, $\Delta F / F_{\text {src }}=0.9952, S_{\text {cent }}=0.51$ mas and $\Delta \tau=86.1 \mathrm{~s}$.

Figure 1 shows color-indexed $\delta P_{\text {tot }}, \delta \Delta P, \delta r_{\text {tot }}, \delta S_{\text {cent }}$ and $\delta \Delta \tau$ which are the deviations of the practical observables from those of the Schwarzschild black hole. It is found that the absolute values of the deviations $\delta P_{\text {tot }}$ and $\delta \Delta P$ range from a few nanoarcsecond (nas) to several tens of nas and $\left|\delta S_{\text {cent }}\right|$ is less than 10 nas, all of which are far beyond the current capability of measurement. After the two lensed images might be able to resolve given their angular separation $P_{\text {tot }} \approx 1.48$ mas, the deviations of the total flux $\Delta r_{\text {tot }}$ and the differential time delay $\delta \Delta \tau$ are respectively no more than 

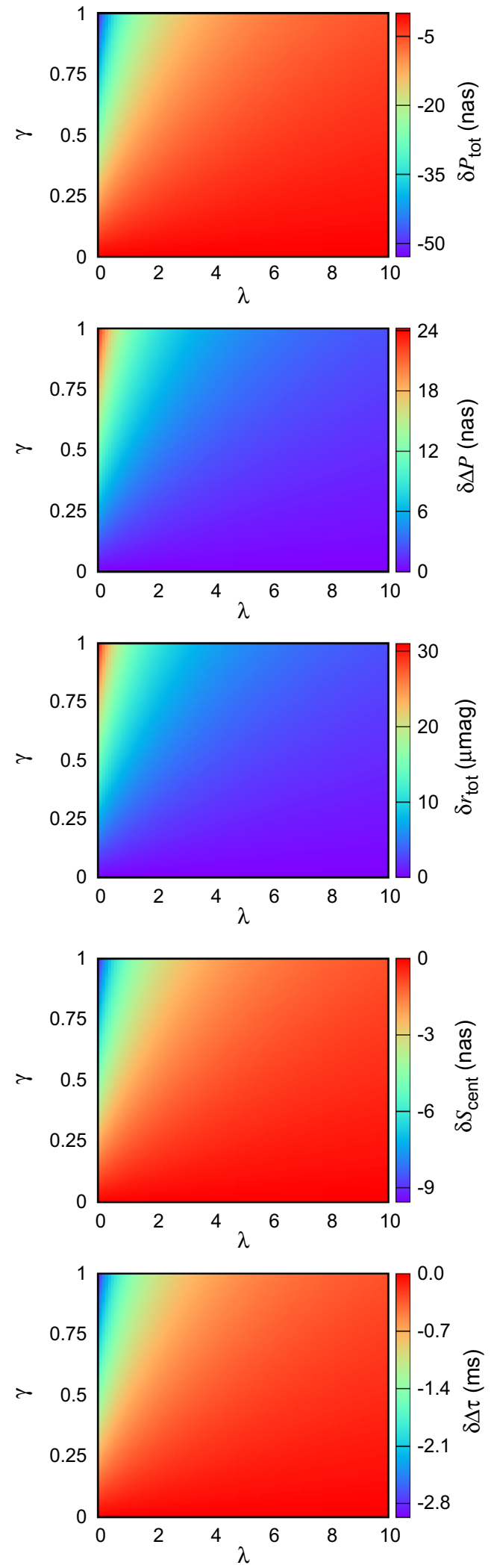

Fig. 1 Color-indexed deviations of practical observables given by the renormalization group improved Schwarzschild black hole from those of the Schwarzschild one in the weak deflection lensing for Sgr A*. From top to bottom, they are $\delta P_{\text {tot }}, \delta \Delta P, \Delta r_{\text {tot }}, \delta S_{\text {cent }}$ and $\delta \Delta \tau$ when $\beta=0.5$
31 micro-mag ( $\mu \mathrm{mag}$ ) and -3 millisecond $(\mathrm{ms})$, neither of which are close to the ability of the present technology since there are no enough photometric and temporal resolutions in the astronomical observations for stars orbiting Sgr A* [88]. Therefore, it is not feasible to test and distinguish the renormalization group improved Schwarzschild black hole by the weak deflection gravitational lensing in the near future.

\section{Strong deflection lensing}

In the strong deflection gravitational lensing, the closet approach distance of the light ray $r_{0}$ is very close to the gravitational radius of the lens, making the bending angle grow and eventually diverge. Thus, a photon can go around the lens for at least one loop if its bending angle is more than $2 \pi$ and then reach the observer, which can never appear in the weak deflection lensing.

\subsection{Strong deflection limit and observables}

The method of strong deflection limit [58] is an analytic scheme to handle the divergence of the bending angle. Its fundamental idea is to expand the bending angle according to the small difference between the impact parameter of the light ray $u$ and its value at the photon sphere. The photon sphere can be defined as the innermost circular orbit for a photon in this work and its radius $r_{m}$ is the biggest root of the equation $[57,95]$

$\frac{C^{\prime}(r)}{C(r)}=\frac{A^{\prime}(r)}{A(r)}$,

where' means derivative with respect to $r$ once. The top panel of Fig. 2 shows dimensionless $x_{m}$ which is rescaled by $r_{m}=x_{m} m_{\bullet}$. The renormalization group improvement makes the radius of the photon sphere shrink compared to its value $x_{m}=3$ for the Schwarzschild black hole. It is similar with the Hayward and non-minimal Einstein-YangMills black holes [73,76], while the modified Hayward and Lee-Wick black holes might have bigger or smaller photon spheres depending on their model parameters [74,75]. After such an expansion, the bending angle in the strong deflection limit can be found as [58]

$\hat{\alpha}(\vartheta)=-\bar{a} \log \left(\frac{\vartheta D_{\mathrm{OL}}}{u_{m}}-1\right)+\bar{b}+\mathcal{O}\left[\left(u-u_{m}\right) \log \left(u-u_{m}\right)\right]$,

where the impact parameter $u$ satisfies $C\left(r_{0}\right)=u^{2} A\left(r_{0}\right)$. Hereafter, the subscript $m$ denotes that a quantity is evaluated at the radius of the photon sphere $r=r_{m}$, such as the impact parameter at the photon sphere $u_{m}$ holding the relation of 

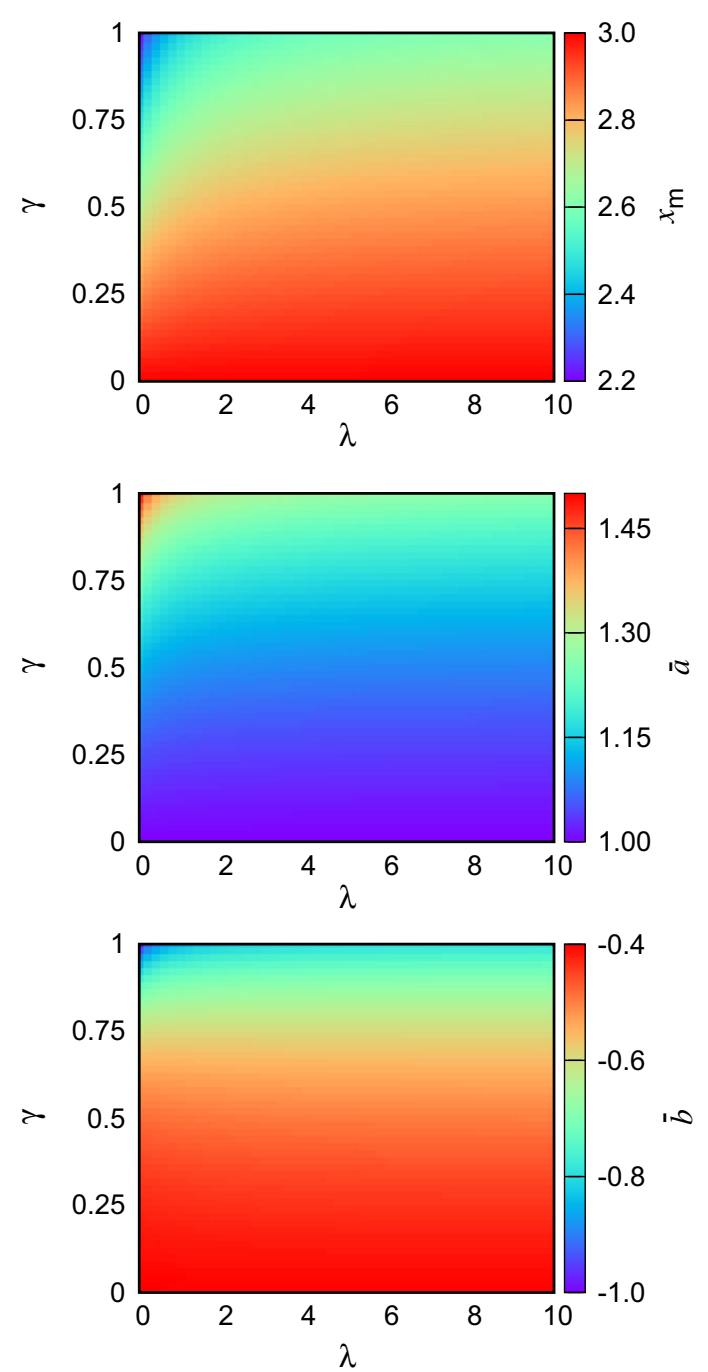

Fig. 2 From top to bottom, color-indexed $x_{m}, \bar{a}$ and $\bar{b}$ are shown. For the Schwarzschild black hole, their values are $x_{m}=3, \bar{a}=1$ and $\bar{b}=-0.4002$

$C\left(r_{m}\right)=u_{m}^{2} A\left(r_{m}\right)$. The coefficients of the bending angle in the strong deflection limit can be found as [58]

$\bar{a}=\frac{R_{m}}{2 \sqrt{\Gamma_{m}}}$,

$\bar{b}=-\pi+b_{R}+\bar{a} \ln \left(\frac{2 \Gamma_{m}}{A_{m}}\right)$,

where

$$
\begin{aligned}
\Gamma_{m} & =\frac{C_{m}\left(1-A_{m}\right)^{2}\left(A_{m} C_{m}^{\prime \prime}-C_{m} A_{m}^{\prime \prime}\right)}{2 A_{m}^{2} C_{m}^{\prime 2}} \\
R_{m} & =\frac{2\left(1-A_{m}\right) \sqrt{A_{m} B_{m}}}{A_{m}^{\prime} \sqrt{C_{m}}} \\
b_{R} & =\int_{0}^{1}\left[\frac{2\left(1-A_{m}\right) \sqrt{A(z) B(z)}}{A^{\prime}(z) C(z) \sqrt{\frac{A_{m}}{C_{m}}-\frac{A(z)}{C(z)}}}-\frac{R_{m}}{z \sqrt{\Gamma_{m}}}\right] \mathrm{d} z,
\end{aligned}
$$

and $z$ is a variable defined as

$z=\frac{A(r)-A_{m}}{1-A_{m}}$.

Here, " means taking derivative against $r$ twice. The middle and bottom panels of Fig. 2 show $\bar{a}$ and $\bar{b}$ which have values $\bar{a}=1$ and $\bar{b}=-0.4002$ for the Schwarzschild black hole. Both of them are more sensitively dependent on $\gamma$, while the increment of the quantum correction $\gamma$ for a given $\lambda$ makes $\bar{a}$ bigger but cause $\bar{b}$ to be smaller. These trends of $\bar{a}$ and $\bar{b}$ with respect to the quantum correction are similar with those for the Bardeen and Hayward black holes [70,73].

We consider a nearly collinear configuration of the source, the lens and the observer in which the source and the observer are sufficiently far from the lens. Such a configuration allows the lens Eq. (11) to be reduced as [96]

$\mathcal{B}=\vartheta-\frac{D_{\mathrm{LS}}}{D_{\mathrm{OS}}}[\hat{\alpha}(\vartheta)-2 n \pi], \quad n \in \mathbb{Z}^{+}$

in which the smallness of $\mathcal{B}, \vartheta$ and $\hat{\alpha}(\vartheta)-2 n \pi$ are ensured by the nearly collinear alignment. The integer $n$ gives the number of loops of the photon moving around the lens, which forms the relativistic images [53].

For a source with changing brightness, the differential time delay between its two relativistic images can be obtained by the total time span in the following form [92]

$T=\tilde{T}\left(r_{0}\right)-\int_{D_{\mathrm{OL}}}^{\infty}\left|\frac{\mathrm{d} t}{\mathrm{~d} r}\right| \mathrm{d} r-\int_{D_{\mathrm{LS}}}^{\infty}\left|\frac{\mathrm{d} t}{\mathrm{~d} r}\right| \mathrm{d} r$.

Since the source and the observer are far from the lens, the second and third terms in the above equation can be easily worked out by means of the approximation for the weak deflection lensing. The first term originates from the strong deflection lensing, depending on the closet approach $r_{0}$ that

$\tilde{T}\left(r_{0}\right)=\int_{r_{0}}^{\infty} 2\left|\frac{\mathrm{d} t}{\mathrm{~d} r}\right| \mathrm{d} r$.

The method of the strong deflection limit can also be employed to expand such an integral as [92]

$\tilde{T}(u)=-\tilde{a} \ln \left(\frac{u}{u_{m}}-1\right)+\tilde{b}+\mathcal{O}\left[\left(u-u_{m}\right) \log \left(u-u_{m}\right)\right]$,

where $\tilde{a}$ and $\tilde{b}$ are its coefficients of the strong deflection limit for the time span and $\tilde{a}=\bar{a} u_{m}$ for the renormalization group improved Schwarzschild black hole.

With the bending angle in the strong deflection limit and the lens equation reduced from the nearly collinear alignment, we are able to find out observables of the strong deflec- 
tion lensing, including the apparent radius of the photon sphere $\theta_{\infty}$, the angular separation between the first relativistic image and other packed images $s$ and their brightness difference $\Delta m$, which are [58]

$$
\begin{aligned}
\theta_{\infty} & =\frac{u_{m}}{D_{\mathrm{OL}}}, \\
s & =\theta_{\infty} \exp \left(\frac{\bar{b}}{\bar{a}}-\frac{2 \pi}{\bar{a}}\right), \\
\Delta m & =2.5 \log _{10}\left[\exp \left(\frac{2 \pi}{\bar{a}}\right)\right],
\end{aligned}
$$

as well as the differential delay between the first and second relativistic images $\Delta T_{2,1}$ and the ratio of its correction $\eta_{2,1}$, which are [92]

$$
\begin{aligned}
\Delta T_{2,1} & =\Delta T_{2,1}^{0}+\Delta T_{2,1}^{1}, \\
\eta_{2,1} & =\frac{\Delta T_{2,1}^{1}}{\Delta T_{2,1}},
\end{aligned}
$$

with

$$
\begin{aligned}
\Delta T_{2,1}^{0}= & 2 \pi u_{m}, \\
\Delta T_{2,1}^{1}= & 2 \sqrt{\frac{B_{m}}{A_{m}}} \sqrt{\frac{u_{m}}{c_{m}}} \exp \left(\frac{\bar{b}}{\bar{a}}\right) \\
\times & {\left[\exp \left(-\frac{\pi}{\bar{a}}\right)-\exp \left(-\frac{2 \pi}{\bar{a}}\right)\right] }
\end{aligned}
$$

and

$c_{m}=\Gamma_{m} \sqrt{\frac{A_{m}}{C_{m}^{3}}} \frac{C_{m}^{\prime 2}}{2\left(1-A_{m}\right)^{2}}$.

Because $\Delta T_{2,1}^{0}$ is proportional to $u_{m}$, it can not tell new insight and extra information would be given by $\Delta T_{2,1}^{1}$.

For the observables of the strong deflection lensing by the renormalization group improved Schwarzschild black hole, their deviations from those of the Schwarzschild black hole in GR can be demonstrated as

$$
\begin{aligned}
\delta \theta_{\infty} & =\theta_{\infty}-\theta_{\infty}(\lambda=\gamma=0), \\
\delta s & =s-s(\lambda=\gamma=0), \\
\delta \Delta m & =\Delta m-\Delta m(\lambda=\gamma=0), \\
\delta \Delta T_{2,1} & =\Delta T_{2,1}-\Delta T_{2,1}(\lambda=\gamma=0), \\
\delta \eta_{2,1} & =\eta_{2,1}-\eta_{2,1}(\lambda=\gamma=0) .
\end{aligned}
$$

\subsection{Example for Sgr A*}

Taking Sgr A* as the lens with $M=4.28 \times 10^{6} M_{\odot}$ and $D_{\mathrm{OL}}=8.32 \mathrm{kpc}$ [94] for the strong deflection lensing, we can estimate its observables. In the case that $\mathrm{Sgr} \mathrm{A} *$ is assumed to be a Schwarzschild black hole in GR, these observables are $\theta_{\infty}=26.4 \mu \mathrm{as}, s=33.0 \mathrm{nas}, \Delta m=6.8 \mathrm{mag}, \Delta T_{2,1}=11.6$ $\min$ and $\eta_{2,1}=1.5 \%$.
Figure 3 shows color-indexed deviations of the observables given by the renormalization group improved Schwarzschild black hole from those of the Schwarzschild black hole in GR, i.e., $\delta \theta_{\infty}, \delta s, \delta \Delta m, \delta \Delta T_{2,1}$ and $\delta \eta_{2,1}$. The existence of the renormalization group improvement can shrink the apparent radius of the photon sphere by nearly 3.9 $\mu$ as in the most significant situation, which is within the ability of EHT [7]. For the relativistic images, such a quantum correction would enlarge their separation $s$ and ratio of time delay components $\eta_{2,1}$; meanwhile, it would reduce their brightness difference $\Delta m$ and differential time delay $\Delta T_{2,1}$. However, the angular resolution demanded to separate the relativistic images is far beyond the present technology, making it impossible to detect these observables, not to mention their deviations from those of the Schwarzschild black hole. Therefore, it is theoretically possible to distinguish the renormalization group improved Schwarzschild black hole from the Schwarzschild black hole only by measuring the apparent size of the photon sphere (shadow) of Sgr A*, whereas any solid conclusion of detection has to consider effects on the shadow caused by the spin of Sgr A* and the highly complicated general relativistic magnetohydrodynamics (GRMHD) of plasma around $\mathrm{Sgr} \mathrm{A}^{*}$, which are beyond the scope of this work.

\subsection{Example for M87*}

Following the same approach, we can find the observables in the strong deflection lensing for M87* with mass $m_{\bullet}=$ $6.5 \times 10^{9} M_{\odot}$ and distance $D_{\mathrm{OL}}=16.9 \mathrm{Mpc}$ [12]. Based on Eqs. (118)-(122), we find the observables for M87* can be obtained by the following scaling relations:

$$
\begin{aligned}
\theta_{\infty, \mathrm{M} 87 *} & =\frac{m_{\bullet, \mathrm{M} 87 *}}{m_{\bullet, \mathrm{SgrA} *}} \frac{D_{\mathrm{OL}, \mathrm{SgrA} *}}{D_{\mathrm{OL}, \mathrm{M} 87 *}} \theta_{\infty, \mathrm{SgrA} *} \\
& =0.7484 \theta_{\infty, \mathrm{SgrA} *}, \\
s_{\mathrm{M} 87 *} & =0.7484 s_{\mathrm{SgrA} *}, \\
\Delta m_{\mathrm{M} 87 *} & =\Delta m_{\mathrm{SgrA} *}, \\
\Delta T_{2,1, \mathrm{M} 87 *} & =\frac{m_{\bullet, \mathrm{M} 87 *}}{m_{\bullet, \mathrm{SgrA} *}} \Delta T_{2,1, \mathrm{SgrA} *} \\
& =1.519 \times 10^{3} \Delta T_{2,1, \mathrm{SgrA} *}, \\
\eta_{2,1, \mathrm{M} 87 *} & =\eta_{2,1, \mathrm{SgrA} *} .
\end{aligned}
$$

They will not change the patterns in Fig. 3 but the ranges of these deviations. As a reference, if assumed to be a Schwarzschild black hole, the observables for M87* in the strong deflection lensing are $\theta_{\infty}=19.7 \mu \mathrm{as}, s=24.7$ nas, $\Delta m=6.8 \mathrm{mag}, \Delta T_{2,1}=294 \mathrm{hr}$ and $\eta_{2,1}=1.5 \%$ in which the values of $\Delta m$ and $\eta_{2,1}$ remain unchanged with respect to those for Sgr A*.

We find that, among the deviations due to the renormalization group improvement for M87*, $\delta \theta_{\infty}, \delta s$ and $\delta \Delta T_{2,1}$ 

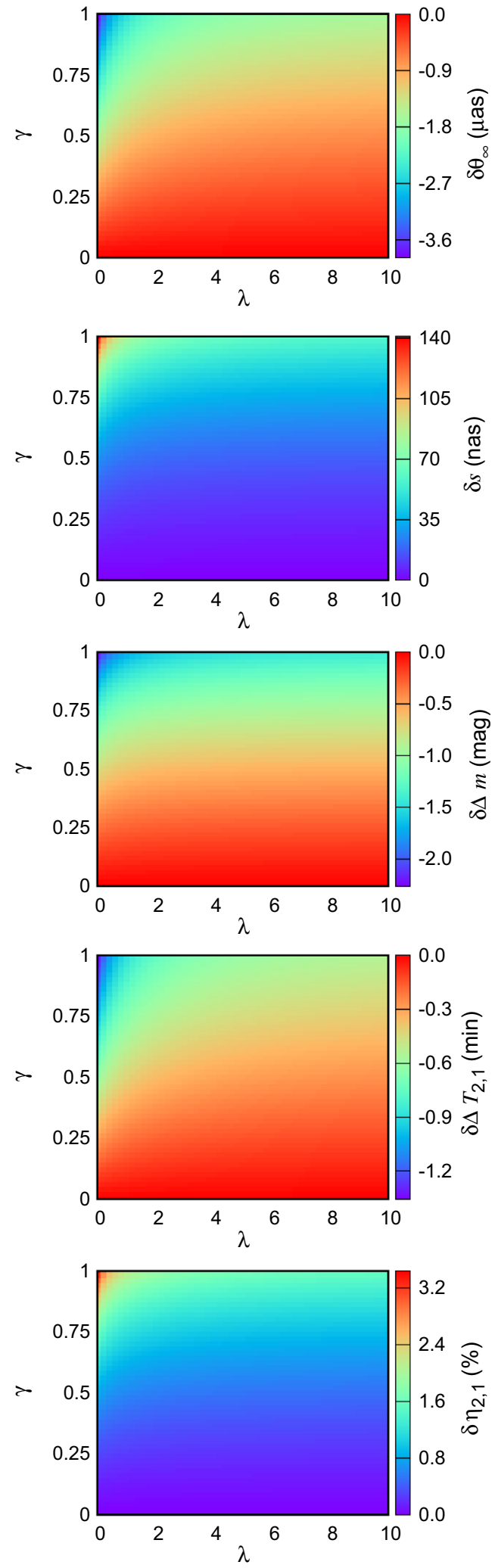

Fig. 3 Color-indexed deviations of observables in the strong deflection lensing for the renormalization group improvement Schwarzschild black hole from those of the Schwarzschild one for Sgr A*. From top to bottom, they are $\delta \theta_{\infty}, \delta s, \delta \Delta m, \delta \Delta T_{2,1}$ and $\delta \eta_{2,1}$ can respectively reach $-2.9 \mu$ as, 105 nas and $-34 \mathrm{~h}$, while $\delta \Delta m$ and $\delta \eta_{2,1}$ are unchanged. It is possible to detect such a quantum correction by measuring the deviation of the shadow $\delta \theta_{\infty}$ whereas other deviations associated with the relativistic images are impractical due to shortage of enough angular resolution. We have to emphasize again that it must be very careful when the measurement by EHT [7] is straightforwardly used to constrain the renormalization group improved Schwarzschild black hole discussed here, because the models for estimating properties of M87*'s shadow [12] have two indispensable ingredients: the rotation of a black hole and GRMHD of plasma around it, neither of which are considered in this work. Nevertheless, based on the measured diameter of $42 \pm 3 \mu$ as of M87*'s shadow [7], we can estimate rough and tentative bounds on $\lambda$ and $\gamma$ as $0.2 \leq \lambda \leq 10$ and $0.02 \leq \gamma \leq 0.22$ in the domain $\mathcal{D}$ by making use of rescaled Fig. 3 according to M87*, which, however, are simply hints for this quantum effect and not genuine constraints on it.

\section{Conclusions and discussion}

The weak and strong deflection gravitational lensing by the renormalization group improved Schwarzschild black hole are studied. The bending angle in the weak deflection lensing is much less than 1 , while the angle in the strong deflection lensing is much larger than 1 . Its observables of the weak deflection lensing, such as the positions, magnifications and differential time delay of lensed images, are obtained; the resulting practical observables and their deviations from those of the Schwarzschild black hole are also constructed and analysed. For a source orbiting Sgr A*, it is possible to measure the total angular separation of its lensed images, whereas none of the deviations of the observables are within the reach of the current technology, making such a black hole indistinguishable from the Schwarzschild one by the weak deflection lensing. Its observables of the strong deflection lensing, including the apparent radius of the photon sphere (shadow) together with the angular separation, the brightness difference and differential time delay between the relativistic images, are obtained; their deviations from those of the Schwarzschild black hole are also worked out. Taking Sgr $\mathrm{A}^{*}$ and M87* as the lenses, We find that the current technology can only measure the apparent sizes of the shadows of these two supermassive black holes and their deviations from those of Schwarzschild one. Based on the measured diameter for M87*'s shadow [7], rough and tentative bounds on the renormalization group improvement parameters are estimated as $0.2 \leq \lambda \leq 10$ and $0.02 \leq \gamma \leq 0.22$.

The renormalization group improved Schwarzschild black hole is an irrotational one that is disfavored by EHT for M87* [7]. General relativistic magnetohydrodynamics of plasma 
around the black hole is not included in our study, though it is important to explain the observed asymmetric ring around M87* [11]. These two key ingredients harm direct usage of M87*'s shadow to constrain the renormalization group improved Schwarzschild black hole in a self-consistent way. Therefore, we only provide some hints of its gravitational lensing signals. More sophisticated investigations with inclusion of its spin in the weak [97-113] and strong [114-118] deflection lensing as well as general relativistic magnetohydrodynamics of plasma are indeed needed.

Acknowledgements This work is funded by the National Natural Science Foundation of China (Grant Nos. 11573015 and 11833004) and the Strategic Priority Research Program of Chinese Academy of Sciences (Grant No. XDA15016700).

Data Availability Statement This manuscript has no associated data or the data will not be deposited. [Authors' comment: This paper is a theoretical work and all of the data are adopted by the related references.]

Open Access This article is licensed under a Creative Commons Attribution 4.0 International License, which permits use, sharing, adaptation, distribution and reproduction in any medium or format, as long as you give appropriate credit to the original author(s) and the source, provide a link to the Creative Commons licence, and indicate if changes were made. The images or other third party material in this article are included in the article's Creative Commons licence, unless indicated otherwise in a credit line to the material. If material is not included in the article's Creative Commons licence and your intended use is not permitted by statutory regulation or exceeds the permitted use, you will need to obtain permission directly from the copyright holder. To view a copy of this licence, visit http://creativecomm ons.org/licenses/by/4.0/.

Funded by SCOAP ${ }^{3}$.

\section{References}

1. LIGO Scientific Collaboration and Virgo Collaboration, Phys. Rev. Lett. 116(6), 061102 (2016). https://doi.org/10.1103/ PhysRevLett.116.061102

2. LIGO Scientific Collaboration and Virgo Collaboration, Phys. Rev. X 6(4), 041015 (2016). https://doi.org/10.1103/PhysRevX. 6.041015

3. LIGO Scientific Collaboration and Virgo Collaboration, Phys. Rev. Lett. 116(24), 241103 (2016). https://doi.org/10.1103/ PhysRevLett.116.241103

4. LIGO Scientific Collaboration and Virgo Collaboration, Phys. Rev. Lett. 118(22), 221101 (2017). https://doi.org/10.1103/ PhysRevLett.118.221101

5. LIGO Scientific Collaboration and Virgo Collaboration, Astrophys. J. Lett. 851, L35 (2017). https://doi.org/10.3847/ 2041-8213/aa9f0c

6. LIGO Scientific Collaboration and Virgo Collaboration, Phys. Rev. Lett. 119(14), 141101 (2017). https://doi.org/10.1103/ PhysRevLett.119.141101

7. Event Horizon Telescope Collaboration, Astrophys. J. Lett. 875, L1 (2019). https://doi.org/10.3847/2041-8213/ab0ec7

8. Event Horizon Telescope Collaboration, Astrophys. J. Lett. 875, L2 (2019). https://doi.org/10.3847/2041-8213/ab0c96
9. Event Horizon Telescope Collaboration, Astrophys. J. Lett. 875, L3 (2019). https://doi.org/10.3847/2041-8213/ab0c57

10. Event Horizon Telescope Collaboration, Astrophys. J. Lett. 875, L4. https://doi.org/10.3847/2041-8213/ab0e85

11. Event Horizon Telescope Collaboration, Astrophys. J. Lett. 875, L5 (2019). https://doi.org/10.3847/2041-8213/ab0f43

12. Event Horizon Telescope Collaboration, Astrophys. J. Lett. 875, L6 (2019). https://doi.org/10.3847/2041-8213/ab1141

13. M. Bojowald, Living Rev. Relat. 8, 11 (2005). https://doi.org/10. 1007/lrr-2005-11

14. J. Bardeen, Proceedings of International Conference GR5 (Tbilisi University Press, Tbilisi, 1968), p. 174

15. S.A. Hayward, Phys. Rev. Lett. 96(3), 031103 (2006). https://doi. org/10.1103/PhysRevLett.96.031103

16. C. Bejarano, G.J. Olmo, D. Rubiera-Garcia, Phys. Rev. D 95(6), 064043 (2017). https://doi.org/10.1103/PhysRevD.95.064043

17. C.C. Menchon, G.J. Olmo, D. Rubiera-Garcia, Phys. Rev. D 96(10), 104028 (2017). https://doi.org/10.1103/PhysRevD.96. 104028

18. V.P. Frolov, G.A. Vilkovisky, Phys. Lett. B 106, 307 (1981). https://doi.org/10.1016/0370-2693(81)90542-6

19. M. Ambrus, P. Hájíček, Phys. Rev. D 72(6), 064025 (2005). https://doi.org/10.1103/PhysRevD.72.064025

20. C. Rovelli, F. Vidotto, Int. J. Mod. Phys. D 23(12), 1442026 (2014). https://doi.org/10.1142/S0218271814420267

21. C. Barceló, R. Carballo-Rubio, L.J. Garay, G. Jannes, Class. Quantum Gravity 32(3), 035012 (2015). https://doi.org/10.1088/ 0264-9381/32/3/035012

22. P.O. Mazur, E. Mottola, Proc. Natl. Acad. Sci. USA 101, 9545 (2004). https://doi.org/10.1073/pnas.0402717101

23. M. Visser, D.L. Wiltshire, Class. Quantum Gravity 21, 1135 (2004). https://doi.org/10.1088/0264-9381/21/4/027

24. C. Barceló, S. Liberati, S. Sonego, M. Visser, Phys. Rev. D 77(4), 044032 (2008). https://doi.org/10.1103/PhysRevD.77.044032

25. S.D. Mathur, Class. Quantum Gravity 26(22), 224001 (2009). https://doi.org/10.1088/0264-9381/26/22/224001

26. S.D. Mathur, D. Turton, J. High Energy Phys. 01, 34 (2014). https://doi.org/10.1007/JHEP01(2014)034

27. B. Guo, S. Hampton, S.D. Mathur, J. High Energy Phys. 07, 162 (2018). https://doi.org/10.1007/JHEP07(2018)162

28. R. Carballo-Rubio, F. Di Filippo, S. Liberati, M. Visser, Phys. Rev. D 98(12), 124009 (2018). https://doi.org/10.1103/PhysRevD.98. 124009

29. V. Cardoso, E. Franzin, P. Pani, Phys. Rev. Lett. 116(17), 171101 (2016). https://doi.org/10.1103/PhysRevLett.116.171101

30. V. Cardoso, E. Franzin, P. Pani, Phys. Rev. Lett. 117(8), 089902 (2016). https://doi.org/10.1103/PhysRevLett.117.089902

31. V. Cardoso, P. Pani, Nat. Astron. 1, 586 (2017). https://doi.org/ 10.1038/s41550-017-0225-y

32. S.M. Du, Y. Chen, Phys. Rev. Lett. 121(5), 051105 (2018). https:// doi.org/10.1103/PhysRevLett.121.051105

33. S.B. Giddings, Phys. Rev. D $90(12), 124033$ (2014). https://doi. org/10.1103/PhysRevD.90.124033

34. S.B. Giddings, Class. Quantum Gravity 33(23), 235010 (2016). https://doi.org/10.1088/0264-9381/33/23/235010

35. S.D. Mathur, Fortschritte der Physik 53, 793 (2005). https://doi. org/10.1002/prop.200410203

36. A. Almheiri, D. Marolf, J. Polchinski, J. Sully, J. High Energy Phys. 2, 62 (2013). https://doi.org/10.1007/JHEP02(2013)062

37. S.L. Liebling, C. Palenzuela, Living Rev. Relat. 20, 5 (2017). https://doi.org/10.1007/s41114-017-0007-y

38. V. Cardoso, P. Pani, Living Rev. Relat. 22(1), 4 (2019). https:// doi.org/10.1007/s41114-019-0020-4

39. A. Bonanno, M. Reuter, Phys. Rev. D 62(4), 043008 (2000). https://doi.org/10.1103/PhysRevD.62.043008 
40. R. Yang, Phys. Rev. D 92(8), 084011 (2015). https://doi.org/10. 1103/PhysRevD.92.084011

41. V. Perlick, Living Rev. Relat. 7, 9 (2004). https://doi.org/10. 12942/lrr-2004-9

42. P. Schneider, J. Ehlers, E.E. Falco, Gravitational Lenses (Springer-Verlag, Berlin, 1992). https://doi.org/10.1007/ 978-3-662-03758-4

43. A.O. Petters, H. Levine, J. Wambsganss, Singularity Theory and Gravitational Lensing (Birkhäuser, Basel, 2001). https://doi.org/ 10.1007/978-1-4612-0145-8

44. P. Schneider, C. Kochanek, J. Wambsganss, in Gravitational Lensing: Strong, Weak and Micro, ed. by G. Meylan, P. Jetzer, P. North. Saas-Fee Advanced Courses, vol 33 (Springer, Berlin, Heidelberg, 2006). https://doi.org/10.1007/978-3-540-30310-7

45. K.C. Sahu, J. Anderson, S. Casertano, H.E. Bond, P. Bergeron, E.P. Nelan, L. Pueyo, T.M. Brown, A. Bellini, Z.G. Levay, J. Sokol, aff1, M. Dominik, A. Calamida, N. Kains, M. Livio, Science 356, 1046 (2017). https://doi.org/10.1126/science.aal2879

46. C.R. Keeton, A.O. Petters, Phys. Rev. D 72(10), 104006 (2005). https://doi.org/10.1103/PhysRevD.72.104006

47. C.R. Keeton, A.O. Petters, Phys. Rev. D 73(4), 044024 (2006). https://doi.org/10.1103/PhysRevD.73.044024

48. C.R. Keeton, A.O. Petters, Phys. Rev. D 73(10), 104032 (2006). https://doi.org/10.1103/PhysRevD.73.104032

49. T.E. Collett, L.J. Oldham, R.J. Smith, M.W. Auger, K.B. Westfall, D. Bacon, R.C. Nichol, K.L. Masters, K. Koyama, R. van den Bosch, Science 360, 1342 (2018). https://doi.org/10.1126/ science.aao2469

50. G. Li, X.M. Deng, Ann. Phys. 382, 136 (2017). https://doi.org/ 10.1016/j.aop.2017.05.001

51. W.G. Cao, Y. Xie, Eur. Phys. J. C 78, 191 (2018). https://doi.org/ 10.1140/epjc/s10052-018-5684-5

52. J.L. Synge, Mon. Not. R. Astron. Soc. 131, 463 (1966). https:// doi.org/10.1093/mnras/131.3.463

53. C. Darwin, Proc. R. Soc. Lond. Ser. A 249, 180 (1959). https:// doi.org/10.1098/rspa.1959.0015

54. V. Bozza, Gener. Relat. Gravit. 42, 2269 (2010). https://doi.org/ 10.1007/s10714-010-0988-2

55. P.V.P. Cunha, C.A.R. Herdeiro, Gener. Relat. Gravit. 50, 42 (2018). https://doi.org/10.1007/s10714-018-2361-9

56. K.S. Virbhadra, D. Narasimha, S.M. Chitre, Astron. Astrophys. 337, 1 (1998)

57. K.S. Virbhadra, G.F.R. Ellis, Phys. Rev. D 62(8), 084003 (2000)

58. V. Bozza, Phys. Rev. D 66(10), 103001 (2002). https://doi.org/ 10.1103/PhysRevD.66.103001

59. V. Bozza, Phys. Rev. D 67(10), 103006 (2003). https://doi.org/ 10.1103/PhysRevD.67.103006

60. S.E. Vázquez, E.P. Esteban, Nuovo Cimento B Ser. 119, 489 (2004). https://doi.org/10.1393/ncb/i2004-10121-y

61. A.Y. Bin-Nun, Phys. Rev. D 81(12), 123011 (2010). https://doi. org/10.1103/PhysRevD.81.123011

62. G.N. Gyulchev, I.Z. Stefanov, Phys. Rev. D 87(6), 063005 (2013). https://doi.org/10.1103/PhysRevD.87.063005

63. S.S. Zhao, Y. Xie, J. Cosmol. Astropart. Phys. 07, 007 (2016). https://doi.org/10.1088/1475-7516/2016/07/007

64. X. Lu, F.W. Yang, Y. Xie, Eur. Phys. J. C 76, 357 (2016). https:// doi.org/10.1140/epjc/s10052-016-4218-2

65. S. Chakraborty, S. SenGupta, J. Cosmol. Astropart. Phys. 7, 045 (2017). https://doi.org/10.1088/1475-7516/2017/07/045

66. H. Ghaffarnejad, M.A. Mojahedi, Res. Astron. Astrophys. 17, 052 (2017). https://doi.org/10.1088/1674-4527/17/6/52

67. H. Ghaffarnejad, H. Neyad, M.A. Mojahedi, Astrophys. Space Sci. 346, 497 (2013). https://doi.org/10.1007/ s10509-013-1462-x

68. C. Ding, C. Liu, Y. Xiao, L. Jiang, R.G. Cai, Phys. Rev. D 88(10), 104007 (2013). https://doi.org/10.1103/PhysRevD.88.104007
69. P. Nicolini, A. Smailagic, E. Spallucci, Phys. Lett. B 632(4), 547 (2006). https://doi.org/10.1016/j.physletb.2005.11.004

70. E.F. Eiroa, C.M. Sendra, Class. Quantum Gravity 28(8), 085008 (2011). https://doi.org/10.1088/0264-9381/28/8/085008

71. J. Schee, Z. Stuchlík, J. Cosmol. Astropart. Phys. 6, 048 (2015). https://doi.org/10.1088/1475-7516/2015/06/048

72. H. Ghaffarnejad, H. niad, Int. J. Theor. Phys. 55(3), 1492 (2016). https://doi.org/10.1007/s10773-015-2787-8

73. S.W. Wei, Y.X. Liu, C.E. Fu, Adv. High Energy Phys. 2015, 454217 (2015). https://doi.org/10.1155/2015/454217

74. S.S. Zhao, Y. Xie, Eur. Phys. J. C 77, 272 (2017). https://doi.org/ 10.1140/epjc/s10052-017-4850-5

75. S.S. Zhao, Y. Xie, Phys. Lett. B 774, 357 (2017). https://doi.org/ 10.1016/j.physletb.2017.09.090

76. F.Y. Liu, Y.F. Mai, W.Y. Wu, Y. Xie, Phys. Lett. B 795, 475 (2019). https://doi.org/10.1016/j.physletb.2019.06.052

77. Z. Horváth, L.Á. Gergely, Z. Keresztes, T. Harko, F.S.N. Lobo, Phys. Rev. D 84(8), 083006 (2011). https://doi.org/10.1103/ PhysRevD.84.083006

78. E.F. Eiroa, C.M. Sendra, Phys. Rev. D 86(8), 083009 (2012). https://doi.org/10.1103/PhysRevD.86.083009

79. R.N. Izmailov, R.K. Karimov, E.R. Zhdanov, K.K. Nand i, Mon. Not. R. Astron. Soc. 483(3), 3754 (2019). https://doi.org/10.1093/ mnras/sty 3350

80. X. Lu, Y. Xie, Mod. Phys. Lett. A 34(20), 1950152 (2019). https:// doi.org/10.1142/S0217732319501529

81. X. Pang, J. Jia, Class. Quantum Gravity 36(6), 065012 (2019). https://doi.org/10.1088/1361-6382/ab0512

82. C.Y. Wang, Y.F. Shen, Y. Xie, J. Cosmol. Astropart. Phys. 04, 022 (2019). https://doi.org/10.1088/1475-7516/2019/04/022

83. H.W. Hamber, S. Liu, Phys. Lett. B 357(1-2), 51 (1995). https:// doi.org/10.1016/0370-2693(95)00790-R

84. N.E. Bjerrum-Bohr, J.F. Donoghue, B.R. Holstein, Phys. Rev. D 67(8), 084033 (2003). https://doi.org/10.1103/PhysRevD.67. 084033

85. P. Bargueño, S. Bravo Medina, M. Nowakowski, D. Batic, Europhys. Lett. 117(6), 60006 (2017). https://doi.org/10.1209/ 0295-5075/117/60006

86. A. Held, R. Gold, A. Eichhorn, J. Cosmol. Astropart. Phys. 2019(6), 029 (2019). https://doi.org/10.1088/1475-7516/2019/ 06/029

87. GRAVITY Collaboration, Astron. Astrophys. 602, A94 (2017). https://doi.org/10.1051/0004-6361/201730838

88. GRAVITY Collaboration, Astron. Astrophys. 615, L15 (2018). https://doi.org/10.1051/0004-6361/201833718

89. GRAVITY Collaboration, Astron. Astrophys. 618, L10 (2018). https://doi.org/10.1051/0004-6361/201834294

90. S. Weinberg, Gravitation and Cosmology: principles and Applications of the General Theory of Relativity (Wiley, New York, 1972)

91. S. Refsdal, Mon. Not. R. Astron. Soc. 128, 295 (1964). https:// doi.org/10.1093/mnras/128.4.295

92. V. Bozza, L. Mancini, Gener. Relat. Gravit. 36, 435 (2004). https:// doi.org/10.1023/B:GERG.0000010486.58026.4f

93. K.S. Virbhadra, C.R. Keeton, Phys. Rev. D 77(12), 124014 (2008). https://doi.org/10.1103/PhysRevD.77.124014

94. S. Gillessen, P.M. Plewa, F. Eisenhauer, R. Sari et al., Astrophys. J. 837, 30 (2017). https://doi.org/10.3847/1538-4357/aa5c41

95. C.M. Claudel, K.S. Virbhadra, G.F.R. Ellis, J. Math. Phys. 42, 818 (2001). https://doi.org/10.1063/1.1308507

96. V. Bozza, S. Capozziello, G. Iovane, G. Scarpetta, Gener. Relat. Gravit. 33, 1535 (2001). https://doi.org/10.1023/A: 1012292927358

97. J. Ibanez, Astron. Astrophys. 124, 175 (1983)

98. I. Bray, Phys. Rev. D 34, 367 (1986). https://doi.org/10.1103/ PhysRevD.34.367 
99. S.A. Klioner, Sov. Astron. 35, 523 (1991)

100. J.F. Glicenstein, Astron. Astrophys. 343, 1025 (1999)

101. M. Sereno, F. de Luca, Phys. Rev. D 74(12), 123009 (2006). https://doi.org/10.1103/PhysRevD.74.123009

102. M.C. Werner, A.O. Petters, Phys. Rev. D 76(6), 064024 (2007). https://doi.org/10.1103/PhysRevD.76.064024

103. M. Sereno, F. de Luca, Phys. Rev. D 78(2), 023008 (2008). https:// doi.org/10.1103/PhysRevD.78.023008

104. A.B. Aazami, C.R. Keeton, A.O. Petters, J. Math. Phys. 52(9), 092502 (2011). https://doi.org/10.1063/1.3642614

105. A.B. Aazami, C.R. Keeton, A.O. Petters, J. Math. Phys. 52(10), 102501 (2011). https://doi.org/10.1063/1.3642616

106. G. He, W. Lin, Int. J. Mod. Phys. D 23, 1450031 (2014). https:// doi.org/10.1142/S021827181450031X

107. G. He, C. Jiang, W. Lin, Int. J. Mod. Phys. D 23, 1450079 (2014). https://doi.org/10.1142/S0218271814500795

108. X.M. Deng, Int. J. Mod. Phys. D 24, 1550056 (2015). https://doi. org/10.1142/S021827181550056X

109. G.S. He, W.B. Lin, Res. Astron. Astrophys. 15, 646 (2015). https:// doi.org/10.1088/1674-4527/15/5/003
110. X.M. Deng, Int. J. Mod. Phys. D 25, 1650082 (2016). https://doi. org/10.1142/S0218271816500826

111. G. He, W. Lin, Phys. Rev. D 93(2), 023005 (2016). https://doi. org/10.1103/PhysRevD.93.023005

112. G. He, W. Lin, Phys. Rev. D 94(6), 063011 (2016). https://doi. org/10.1103/PhysRevD.94.063011

113. G. He, W. Lin, Class. Quantum Gravity 34(10), 105006 (2017). https://doi.org/10.1088/1361-6382/aa691d

114. J.M. Bardeen, in Black Holes (Les Astres Occlus), ed. by C. Dewitt, B.S. Dewitt (Gordon and Breach, 1973), pp. 215-239

115. V. Bozza, F. de Luca, G. Scarpetta, M. Sereno, Phys. Rev. D 72(8), 083003 (2005). https://doi.org/10.1103/PhysRevD.72.083003

116. V. Bozza, F. de Luca, G. Scarpetta, Phys. Rev. D 74(6), 063001 (2006). https://doi.org/10.1103/PhysRevD.74.063001

117. V. Bozza, Phys. Rev. D 78(6), 063014 (2008). https://doi.org/10. 1103/PhysRevD.78.063014

118. S.V. Iyer, E.C. Hansen, Phys. Rev. D 80(12), 124023 (2009). https://doi.org/10.1103/PhysRevD.80.124023 